Helgoländer wiss. Meeresunters. 21, 271-291 (1970)

\title{
Tauchuntersuchungen zur Vertikalverteilung der sublitoralen Helgoländer Algenvegetation
}

\author{
K. LÜNING \\ Biologische Anstalt Helgoland (Meeresstation); Helgoland, Deutschland (BRD)
}

\begin{abstract}
Diving investigations on the vertical distribution of sublittoral algal vegetation near Helgoland. The vertical distribution of macroscopic sublittoral algae near the island of Helgoland (North Sea) has been investigated using the Scuba diving tedinique. Laminaria digitata and L. saccbarina occupy the depth range between 0.5 and $1.5 \mathrm{~m}$ (below mean low water of spring tides). The $L$. byperborea forest extends between 1.5 and $4 \mathrm{~m}$ depth. The deepest-growing specimens of $L$. hyperborea have been recorded at $8 \mathrm{~m}$ depth, the deepest algae (encrusting forms) at $15 \mathrm{~m}$. Some species are confined to the upper part of the sublittoral zone (e. g. Chondrus crispus, Dumontia incrassata, Abnfeltia plicata, Polyides rotundus, Furcellaria fastigiata), while others penetrate to the lower part (e. g. Pbycodrys rubens, Delesseria sanguinea, Brongniartella byssoides, Pbyllophora crispa, Bryopsis plumosa).
\end{abstract}

\section{EINLEITUNG}

Die bisherigen Untersuchungen über den Vegetationsaufbau der Helgoländer marinen Algenflora mußten sich aus technischen Gründen auf das Supralitoral, Eulitoral und den obersten, bei extremem Niedrigwasser freifallenden Bereich des Sublitorals beschränken (KuckUCK 1897, Nienburg 1925, Hartog 1959). Über die im Helgoländer Sublitoral vorkommenden Algenarten war man zwar seit Kuckucks Zeiten durch Untersuchung von gedredschtem und angetriebenem Material weitgehend unterrichtet, nicht jedoch über die Tiefenverteilung der einzelnen Arten. NIENBurg (1925) schloß seine Ausführungen über die Vegetationsverhältnisse der Helgoländer sublitoralen Abrasionsterrasse und Geröllzonen mit dem Satz: „Weiter wird man darin erst kommen, wenn man einmal den Tauchapparat in den Dienst der Wissenschaft gestellt hat."

Solange nur der schwerfällige Helmtaucher in das Sublitoral einzudringen vermochte, blieb die Zahl der biologisch arbeitenden Tauchpioniere klein (z. B. GisLeN 1929, 1930, Kitching et al. 1934, KItching 1941, WAern 1952). Erst die technische Entwicklung der modernen Schwimmtauchmethode führte dazu, daß eine ständig wachsende Anzahl von Meeresbiologen das Tauchen als wissenschaftliche Methode verwendete. Die historische Entwicklung des wissenschaftlichen Tauchens wurde von RrEDL (1967) ausführlich dargestellt. Auf meeresbotanischem Gebiet sind mit Hilfe der Schwimmtauchmethode bereits zahlreiche ökologisch und vegetationskundlich 
ausgerichtete Untersuchungen durchgeführt worden (z. B. ERNST 1955, 1959, ALEEM 1956, Morgans 1957, Kornas 1959, Kain 1960, 1962, 1963, 1967, McLean 1962, Lamb \& Zimmermann 1964, Neushul 1965, 1967, Clarke \& Neushul 1967, Michanek 1967, Smith 1967, Larkum et al. 1967, McAllister et al. 1967, Norton 1968). An der Biologischen Anstalt Helgoland konnte ab 1965, seit der Einrichtung

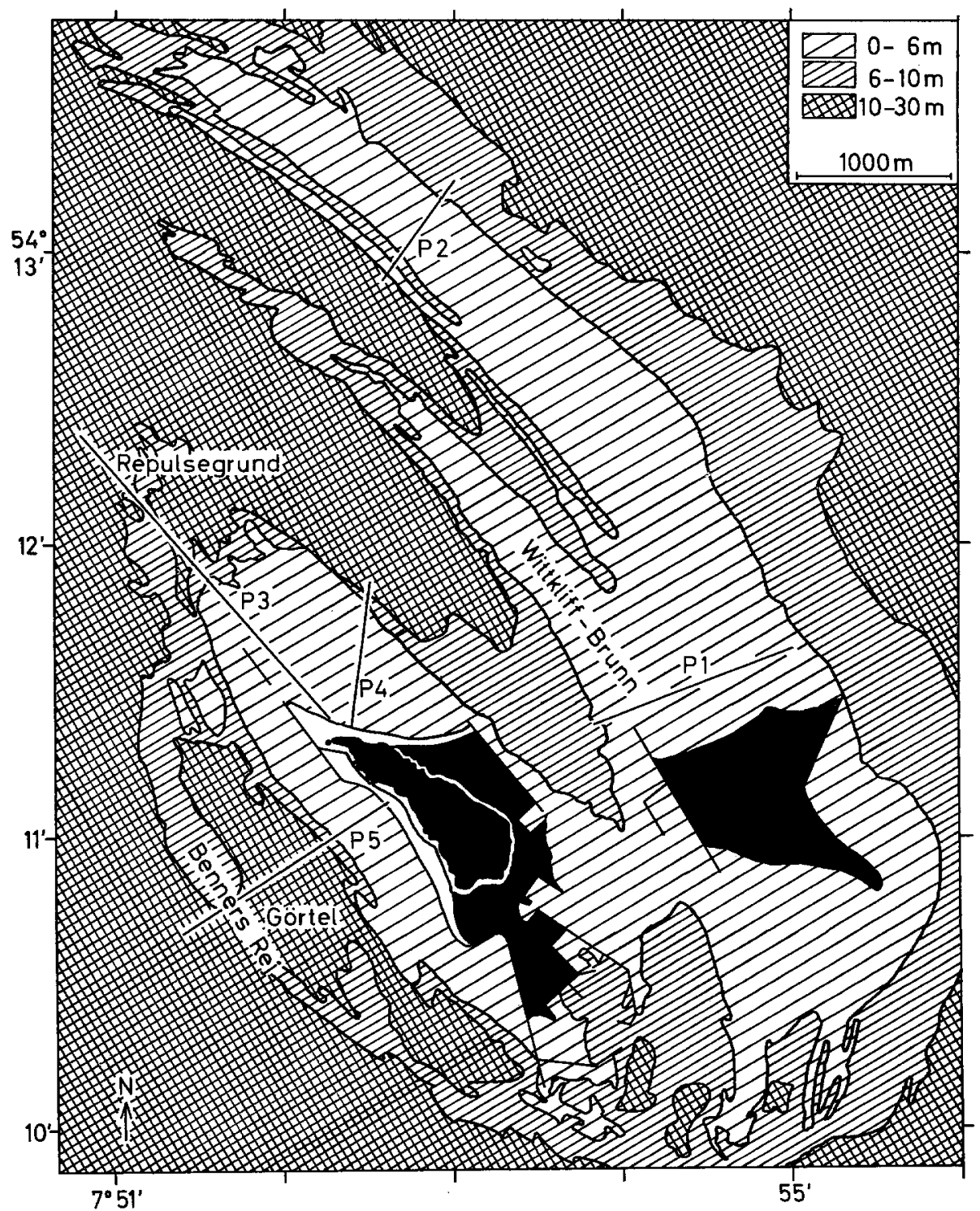

Abb. 1: Karte des Seegebiets um Helgoland (gezeichnet nach Seekarte Nr. 88) mit Tiefenlinien bei $0 \mathrm{~m}, 6 \mathrm{~m}, 10 \mathrm{~m}$ sowie Verlauf der Tauchprofile P 1 bis P 5 
einer Schwimmtauchgruppe, die Tiefenverteilung sublitoraler Algen untersucht werden. Die vorliegende Arbeit faßt die bisherigen Ergebnisse zusammen.

\section{GEBIET, MATERIAL UND METHODEN}

Die Tiefenverteilung der makroskopischen Algenarten wurde auf fünf Tauchprofilen von insgesamt $7200 \mathrm{~m}$ Länge untersucht. Thre Lage ist in den Abbildungen 1 und 2 angegeben. Profil P 3 überquert den Buntsandsteinsockel der Hauptinsel in nordwestlicher Richtung und setzt sich auf den Kreidefelsen des "Repulsegrundes" fort; Profil P 4, in nördlicher Richtung verlaufend, endet in dem Geröllfeld des "Stroms“, zwischen dem Buntsandsteinsockel der Hauptinsel und den nördlichen Dünenklippen.

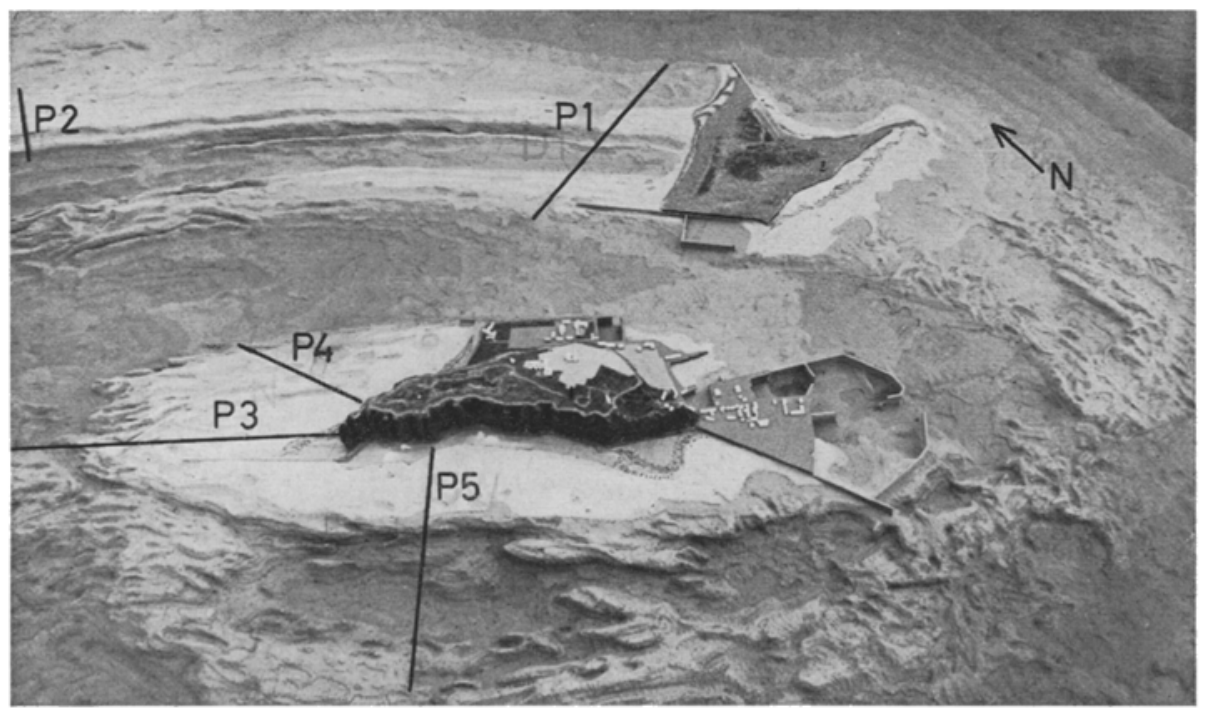

Abb. 2: Relief des untermeerischen Felsgebiets um Helgoland mit Verlauf der Tauchprofile P 1 bis P 5 (Modell im Besitz der Gemeinde Helgoland; Foto: Vahlendieck, Schleswig)

Profil P 5 überquert in südwestlicher Richtung den Buntsandsteinsockel, führt durch das tiefe Geröllffeld des "Görtel" und erreicht die Kreidefelsen des „Benners Rej". Profil P 1 erfaßt die Muschelkalkklippen ("Witt-Kliff-Brunn“) und Kreideklippen nördlich der Düneninsel. Weiter nördlich werden die Kreideklippen noch einmal von Profll P 2 überquert. Die Morphologie und Hydrographie des Helgoländer Seegebietes wurde durch Hagmeier (1930) dargestellt.

Vor Beginn eines jeden Tauchgangs wurde in festgelegter Peilung (Tonnenstrich, Landmarken oder Kompaßkurs) vom Boot aus eine $200 \mathrm{~m}$ lange Perlonleine $(8 \mathrm{~mm}$ stark) versenkt, die mit Farbmarken in 5-m-Abständen markiert und mit Betonklötzen beschwert war (Abb. 3). Nach beendetem Tauchgang wurde am Ende der Profilleine eine Markierungsboje gesetzt, an welcher vor dem nächsten Tauchgang die weitere Verlegung der Profilleine begann. 
Die Tauchausrüstung (Abb. 4) bestand aus Neoprenanzug, Tauchmaske, Flossen, Preßluftlaschen, Lungenautomat, Bleigürtel, Rettungsweste und Messer. Da es sich als zu langwierig erwies, unter Wasser Daten auf Kunststofftafeln zu schreiben, wurde ein Unterwassermikrofon verwendet. Das Kabel führte durch die hohle Sicherheitsleine zum Boot, welches den längs der Profilleine schwimmenden Taucher begleitete (Abb. 3). Die vom Taucher in das Mikrofon gesprochenen Angaben wurden auf einem im Boot installierten, batteriegetriebenen. Tonbandgerät aufgenommen.

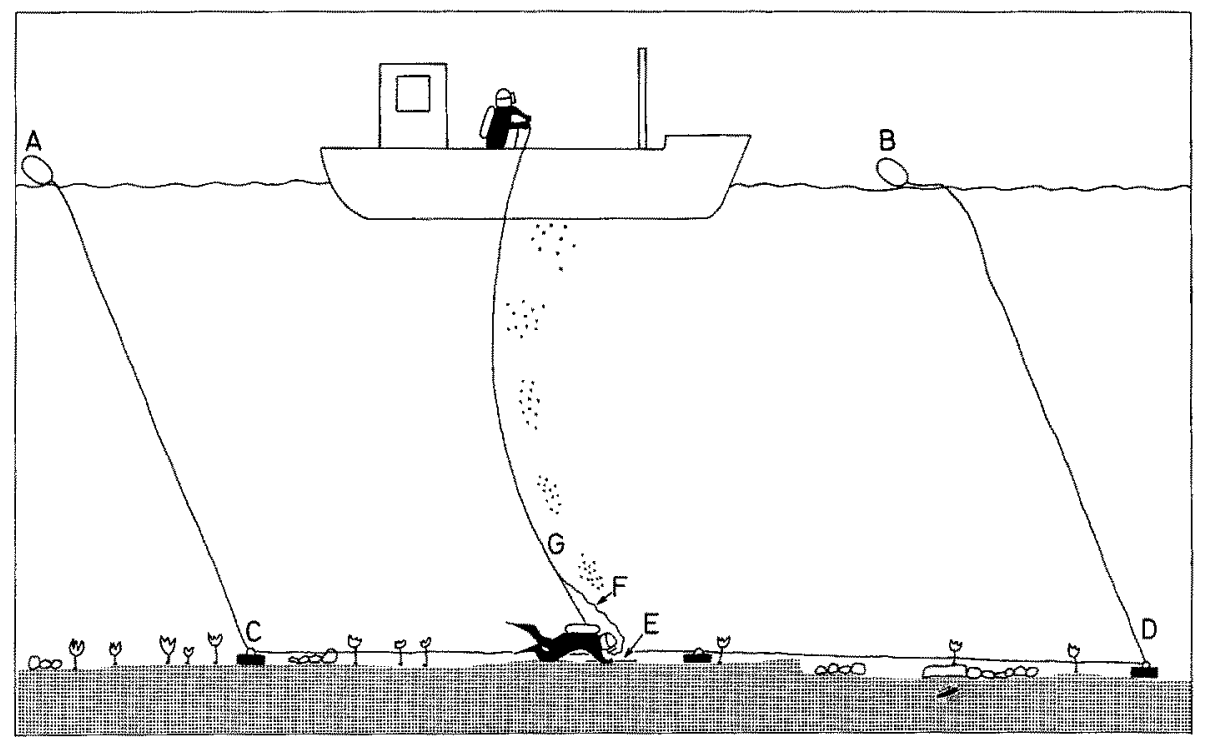

Abb. 3: Schema der Vegetationsaufnahme im Sublitoral. An Boje A wird zum Anfangspunkt C abgetaucht. Die Vegetationsaufnahme wird entlang der $200 \mathrm{~m}$ langen Grundleine mit Hilfe des Probequadrats E durchgeführt. Daten werden über das Kabel F, welches mit der Sicherheitsleine $G$ verbunden ist, auf ein an Bord des Schiffes mitgeführtes Tonbandgerät gesprochen. Nach Erreichung des Endpunkts D wird zur Boje B aufgetaucht

Längs jedes 5-m-Abschnittes der Profilleine wurde das Vorkommen der vegetationsbestimmenden Brauntange (Fucus serratus, Laminaria digitata, L. saccharina, L. byperborea) registriert. Im Tiefenbereich zwischen $+0,2 \mathrm{~m}$ und $-3,0 \mathrm{~m}$ stehen zur Auswertung die Daten von 3795 -m-Abschnitten zur Verfügung. Zur Erfassung kleinerer Algen wurde ein Quadratrahmen $\left(1 \mathrm{~m}^{2}\right)$ mitgefürt, der in $16 \mathrm{Kleinquadra-}$ te aufgeteilt war (Abb. 4). In jedem Kleinquadrat wurde das Vorkommen aller mit bloßem Auge sichtbaren Algenarten erfaßt. Proben von 67 Quadraten wurden in das Labor mitgenommen, mikroskopiert und in Formol fixiert. Wies die zu untersuchende Felspartie oder etwa ein Felsklotz eine kleinere Fläche als $1 \mathrm{~m}^{2}$ auf, so konnten nur acht bzw. vier Kleinquadrate untersucht werden. Insgesamt stehen im Tiefenbereich $z$ wischen $\mathrm{O}$ und $15 \mathrm{~m}$ die Daten von 1232 Kleinquadraten $(25 \times 25 \mathrm{~cm})$ zur Verfügung, die auf Lochkarten übertragen wurden. Die Anzahl der in verschiedenen Tiefenstufen untersuchten Quadrate geht aus Tabelle 1 hervor. 
Tabelle 1

Anzahl der in verschiedenen Tiefenstufen untersuchten Quadrate $(25 \times 25 \mathrm{~cm})$

\begin{tabular}{|lccccccc|}
\hline Tiefenstufe & $0-0,5 \mathrm{~m}$ & $0,5-1 \mathrm{~m}$ & $1-1,5 \mathrm{~m}$ & $1,5-2 \mathrm{~m}$ & $2-3 \mathrm{~m}$ & $3-4 \mathrm{~m}$ & $4-5 \mathrm{~m}$ \\
Anzahl Quadrate & 80 & 136 & 248 & 144 & 120 & 62 & 62 \\
\hline Tiefenstufe & $5-6 \mathrm{~m}$ & $6-7 \mathrm{~m}$ & $7-8 \mathrm{~m}$ & $8-10 \mathrm{~m}$ & $10-12 \mathrm{~m}$ & $12-15 \mathrm{~m}$ \\
Anzahl Quadrate & 82 & 60 & 99 & 64 & 50 & 25 \\
\hline
\end{tabular}

Am Beginn jedes 5-m-Abschnittes der Profilleine und bei jedem Auslegen des Quadratrahmens wurden Tiefe, Uhrzeit und Art des Substrates registriert. Zur Tiefenbestimmung wurde ein Kapillartiefenmesser verwendet, eine oben zugeschmolzene, $75 \mathrm{~cm}$ lange Glasröhre in einer Holzhalterung (Abb. 4). Kontrolleichungen längs einer markierten Tiefenleine zeigten eine gleichbleibende Genauigkeit der Ablesung von

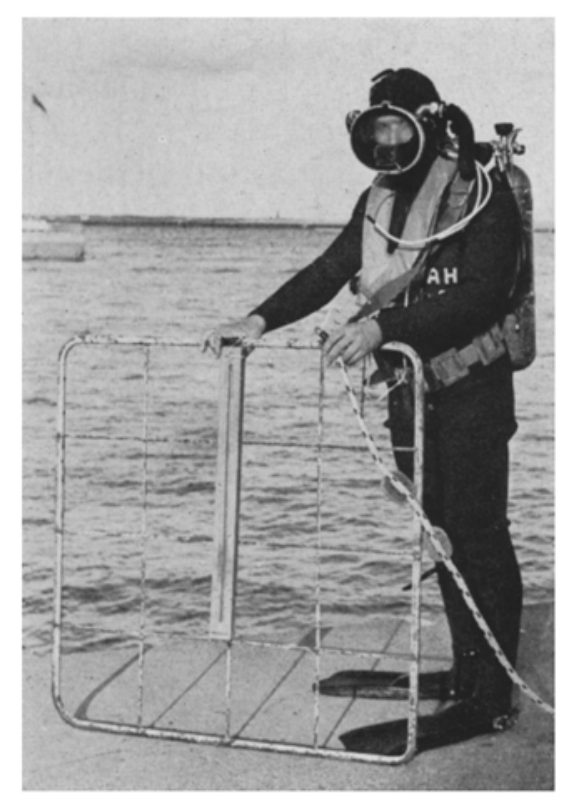

Abb. 4: Schwimmtaucher mit Probequadrat zur Vegetationsaufnahme. Am Quadrat ist ein $0,75 \mathrm{~m}$ langer Kapillartiefenmesser befestigt. Am unteren Rand der Vollgesichtsmaske befindet sich ein Mikrofon, dessen Kabel über den Kopfhörer zur Sicherheitsleine führt

$\pm 0,1 \mathrm{~m}$. Zusätzlich zur jeweiligen Tiefe wurde die Uhrzeit festgehalten, so daß alle Tiefenmessungen mit Hilfe der Aufzeichnungen der Pegelstelle „Helgoland Südhafen“ auf Deutsches Kartennull ( $=$ mittleres Springniedrigwasser $=$ M.S.N.W.) reduziert werden konnten. Der mittlere Tidenhub beträgt bei Helgoland 2,3 m, der Unterschied zwischen mittlerem Niedrigwasser und mittlerem Springniedrigwasser 0,2 m. Das tiefste Niedrigwasser seit 1952 wurde am 15. 3. 1964 mit 2,01 m unter M.S.N.W. beobachtet. 
Hinsichtlich der Art des Substrates wurde zwischen anstehendem Fels (Buntsandstein, Muschelkalk, Kreide), Steingeröll, Schill und Sand unterschieden. Verstreut fanden sich in den tiefen Geröllfeldern auch meterhohe Granitfindlinge.

Die in dieser Arbeit dargestellten Tauchprofile wurden von 1967 bis 1968 während der Sommermonate untersucht. Weitere Tauchgänge erfolgten ohne Verlegung einer Profilleine und hatten eine ausgedehnte Flächensuche zum Ziel, um auch seltene Arten zu erfassen. Die Nomenklatur folgt der "Check-list of British marine algaesecond revision" von PARke \& Dixon (1968). In Tabelle 2 sind die von 1965 bis 1970 bei Tauchgängen gefundenen 66 Algenarten bzw. nicht weiter identifizierten Gattungen alphabetisch aufgeführt. Die bisher üblichen Synonyme sind ebenfalls angegeben. Die Kennzeichnung physiognomischer Algentypen wurde nach einer Aufstellung von SchweNkE (1964) durchgeführt. Danach wird unterschieden zwischen (1) Brauntangen (wie Laminaria, Fucus), (2) Feinalgen (z. B. Busch- und Blattbuschalgen, wie Cladophora, Delesseria, Chondrus; Schlauch- und Schnuralgen, wie Chorda; Flächenalgen wie Ulva; Fadenalgen, wie Chaetomorpha), (3) Krusten- und Kalkalgen, wie Lithotbamnion, Corallina, (4) (mikroskopische) Kleinalgen (Kleinbenthos, Kleinepiphyten, endophytische und endozoische Formen).

Für die vorliegende Arbeit gelten folgende Beschränkungen:

(1) Der Tiefenbereich, in dem eine Art gefunden wurde, bezieht sich auf das Gebiet des sublitoralen Helgoländer Felssockels, nicht auf Standorte an Molen, an denen Tiefenarten in geringeren Tiefen vorkommen als auf horizontal verlaufenden Felsklippen. (2) Es werden, mit einer Ausnahme, nur makroskopische Algenarten aufgeführt, die vom Taucher erkannt werden konnten. Als einziger Vertreter der mikroskopischen Kleinalgen wird Audowinella membranacea berücksichtigt. Diese Rotalge durchzieht mit ihren Basallagern die Wände von Hydroidpolypen, verleiht diesen eine rötliche Färbung und fällt daher dem Taucher auf. (3) Selten gefundene Algen, Arten, die in weniger als acht der untersuchten 1232 Kleinquadrate vorkamen oder, unabhängig von Tauchprofilen, erst nach ausgedehnter Flächensuche gefunden wurden, sind in Tabelle 2 mitaufgeführt. Die Tiefenangaben können in diesen Fällen jedoch nicht als repräsentativ für den Vorkommensbereich angesehen werden. Bei zukünftigen Tauchgängen werden möglicherweise noch weitere seltene Arten im Helgoländer Sublitoral entdeckt werden. (4) Von Krustenalgen werden nur einige Gattungen der Rbodopbyceae aufgeführt, obwohl auch Vertreter der Pbaeophyceae und Cbloropbyceae gefunden wurden. Die Identifizierung und Tiefenverteilung dieser taxonomisch schwierigen Gruppe muß, wie auch die Untersuchung der mikroskopischen Kleinalgen, späteren Bearbeitungen vorbehalten bleiben.

\section{ERGEBNISSE}

\section{Die Vertikalverteilung der Laminaria-Arten}

Im Tiefenbereich zwischen 0 und $4 \mathrm{~m}$ wächst auf dem stabilen Felssubstrat eine weitgehend geschlossene Vegetation von Laminaria-Arten. Von der Uppigkeit dieser Vegetation erhält man, ohne tauchen zu müssen, bei extremen Niedrigwasserständen, 


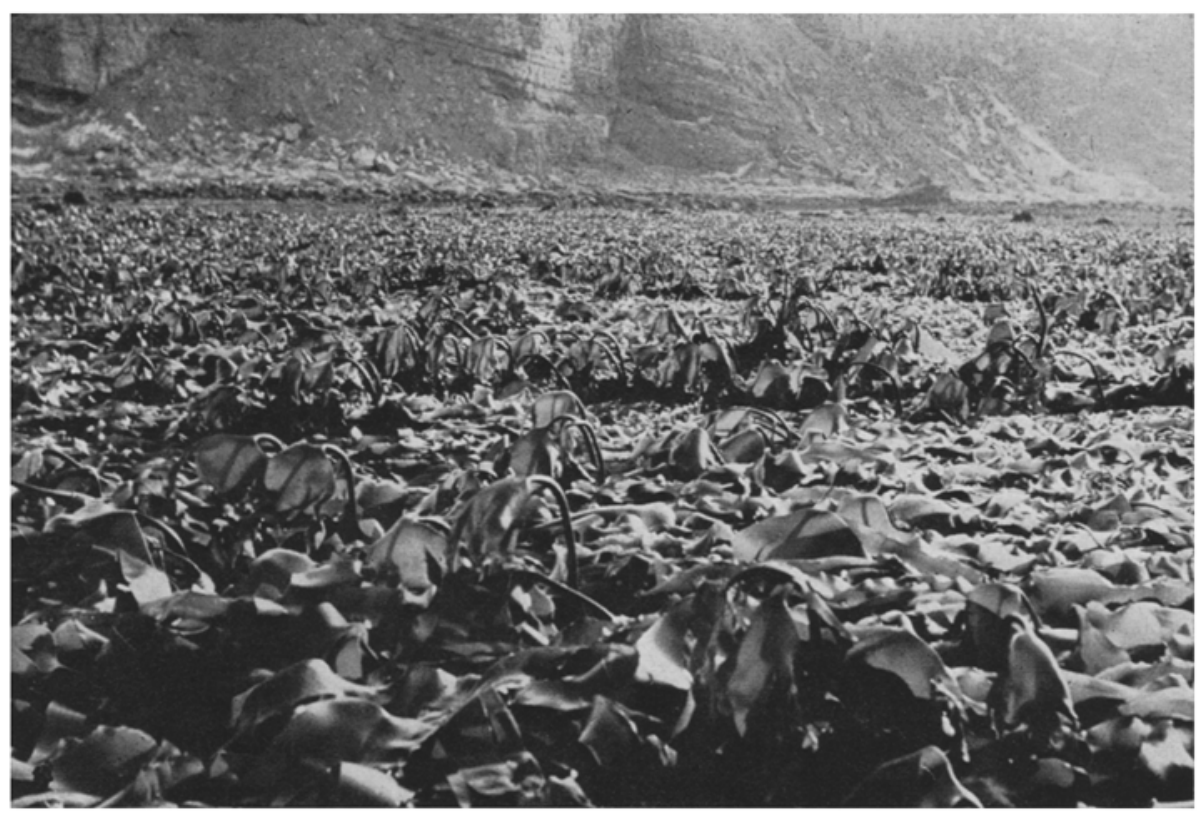

Abb. 5: Bestände von Laminaria digitata (ältere Exemplare aufragend) und L. saccharina (niederliegend). Nordostwatt bei niedrigem Wasserstand

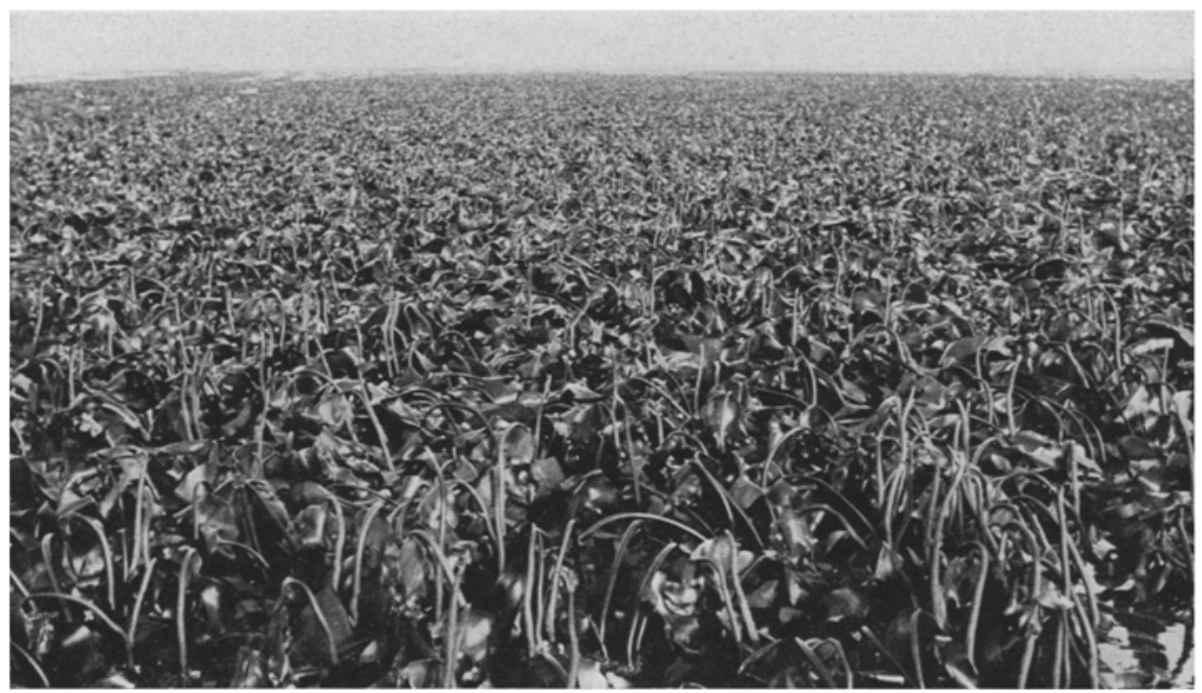

Abb. 6: Geschlossener Bestand von Laminaria byperborea (sogenannter Laminarienwald). Blick auf die Nathurnklippen (etwa im Bereich des Sektors zwischen P 3 und P 4 in Abb. 1) bei niedrigem Wasserstand $(1,5 \mathrm{~m}$ unter 0$)$ 
die im zeitigen Frühjahr bei anhaltendem Ostwind auftreten, einen guten Eindruck. Abbildung 5 zeigt geschlossene Bestände von Laminaria digitata; inselartig eingestreut sind niederliegende Bestände von Laminaria saccharina. Bei sehr niedrigen Wasserständen, die in mehrjährigem Abstand auftreten, fallen auch die obersten Bestände von Laminaria byperborea trocken und erfrieren dabei weitgehend (Abb. 6).

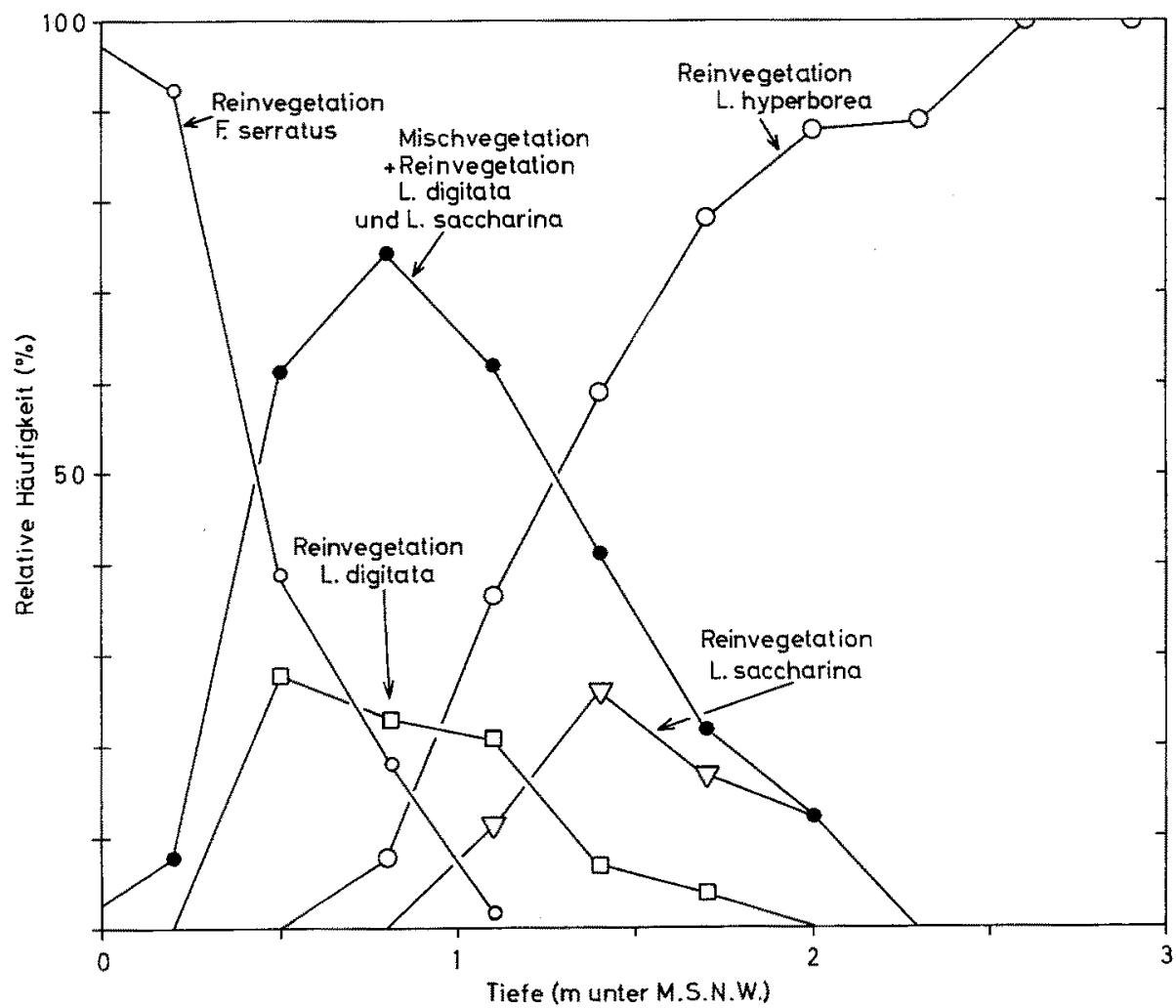

Abb. 7: Relative Häufigkeit von geschlossenen Brauntangbeständen (Fucus serratus, Laminaria digitata, L. saccharina, L. byperborea) in Abhängigkeit von der Wassertiefe (Meter unter mittlerem Springniedrigwasser)

Abbildung 7 zeigt die Tiefenverteilung von Reinbeständen der Arten Fucus serratus, Laminaria digitata, L. saccharina, L. byperborea sowie von Rein- und Mischbeständen der Arten $L$. digitata und $L$. saccharina im Tiefenbereich zwischen $O$ und $3 \mathrm{~m}$. Die Gesamtanzahl von 379 in diesem Bereich untersuchten 5-m-Abschnitten der Profilleine wurde in elf Tiefenstufen aufgegliedert. Die Tiefenverteilung der verschiedenen Bestände (charakterisiert durch das Auftreten der einzelnen Arten längs eines 5-m-Abschnitts) wurde als prozentualer Anteil an der Gesamtzahl der in jeder Tiefenstufe untersuchten 5-m-Abschnitte berechnet.

Die Vegetation mit Fucus serratus wird unterhalb von $0 \mathrm{~m}$ in steigendem Ausmaß durch die Vegetation von Laminaria digitata und L. saccharina ersetzt, die zwi- 
schen 0,4 und 1,3 m Tiefe in Form von Rein- und Mischbeständen in mehr als 50\% aller untersuchten 5 -m-Abschnitte auftrat. An der oberen Grenze des genannten Tiefenbereichs wachsen Reinbestände von Laminaria digitata, an der unteren Grenze Reinbestände von $L$. saccharina, während Mischbestände im mittleren Bereich vorherrschen. Die tiefsten mehrjährigen Vertreter von $L$. digitata wurden in $1,9 \mathrm{~m}$ Tiefe, von $L$. saccharina in $2,9 \mathrm{~m}$ Tiefe angetroffen. Wenige Monate alte Sporophyten von $L$. saccharina, die sich von den mehrjährigen Pftanzen durch ein sehr schmales Phylloid unterscheiden, wurden im Sommer auch zwischen 2,9 und $9 \mathrm{~m}$ Tiefe gefunden.

Die obersten Vertreter von Laminaria byperborea in $0,3 \mathrm{~m}$ sind kurzstielig (Stiellänge 10-20 cm). Mit der Tiefe nimmt auch die Stiellänge zu. An der unteren Grenze der geschlossenen Vegetation von L. byperborea, des "Laminarienwaldes" (in $4 \mathrm{~m}$ Tiefe), sind Pflanzen mit einer Stiellänge von 1,5 m keine Seltenheit. Die Populationsdichte verringert sich innerhalb des Laminarienwaldes mit zunehmender Tiefe (im Mittel von 27 Individuen pro $\mathrm{m}^{2}$ in $2 \mathrm{~m}$ Tiefe auf 10 Individuen pro $\mathrm{m}^{2}$ in $4 \mathrm{~m}$ Tiefe), ebenso die Gesamtfläche aller Phylloide pro Flächeneinheit. Im Mittel werden in $2 \mathrm{~m}$ Tiefe $4,1 \mathrm{~m}^{2}$ Phylloidfläche pro $\mathrm{m}^{2}$ Felsfläche produziert, dagegen nur $1,6 \mathrm{~m}^{2}$ in $4 \mathrm{~m}$ Tiefe (LünING 1969). Bei dieser Tiefe geht der geschlossene Laminarienwald in eine offene Vegetation über. Zwischen vereinzelten langstieligen und an den Stielen reich mit Epiphyten besetzten Exemplaren von L. hyperborea wachsen hier inmitten einer üppigen Busch- und Blattbuschalgenvegetation auch zahlreiche Jungpflanzen von $L$. byperborea. Mit zunehmender Tiefe findet man immer häufiger Exemplare von $L$. byperborea, die mehr oder weniger vollständig von der Bryozoe Membranipora membranacea überwachsen sind. Auch die im Laminarienwald, im Schatten des Phylloiddachs wachsenden kleinen Exemplare von L. byperborea sind häufig stark von der Bryozoe Membranipora membranacea besiedelt, der es offensichtlich leicht gelingt, die infolge Lichtmangels langsam wachsende Alge vollständig zu überziehen. Die tiefsten mehrjährigen Vertreter von $L$. byperborea wurden an allen Profilen bei $8 \mathrm{~m}$ Tiefe gefunden. Jungpflanzen von L. hyperborea können im Sommer in noch etwas größerer Tiefe gedeihen, sie sterben aber offensichtlich in der lichtarmen Jahreszeit, im Winter, unterhalb von $8 \mathrm{~m}$ Tiefe $\mathrm{ab}$.

Die Abbildungen 8 und 9 stellen die Tiefenverteilung der vegetationsbestimmenden Brauntange anhand von Tauchprofilen dar. Profil P 3 (Abb. 8) führt im Nordwesten Helgolands über die aus Buntsandstein bestehende Abrasionsterrasse und über die Kreide des "Repulsegrundes" (Abb. 1). Bis zu einer Entfernung von $500 \mathrm{~m}$ von der Strandlinie herrschen Fucus serratus, Laminaria digitata und L. saccharina vor. Danach folgt bis etwa $1100 \mathrm{~m}$ die geschlossene Vegetation von L. byperborea, der sogenannte Laminarienwald. Der Abhang der Abrasionsterrasse (Abb. 8, 1100 bis $1400 \mathrm{~m}$ ) ist im Norden und Nordosten der Insel stark zerklüftet. Die Zone zwischen Buntsandstein- und Kreidefels (Abb. 8, 1400-1500 m) stellt ein Geröllfeld dar (Buntsandstein- und Kreidebruchstücke sowie Hartgeröll). Auf den anschließenden Kreideklippen des "Repulsegrundes" kommt $L$. byperborea, wie auf Buntsandstein, wieder nur oberhalb von $8 \mathrm{~m}$ Tiefe vor. Anstehender Kreidefels wurde noch in $21 \mathrm{~m}$ Tiefe (Abb. 8, $2300 \mathrm{~m}$ ) gefunden.

Die Abrasionsterrasse im Südwesten der Insel (Abb, 9, $0-430 \mathrm{~m}$ ) ist wesentlich schmaler als im Norden und Nordosten (Abb. 1 und 2). Der westliche Abhang ist 

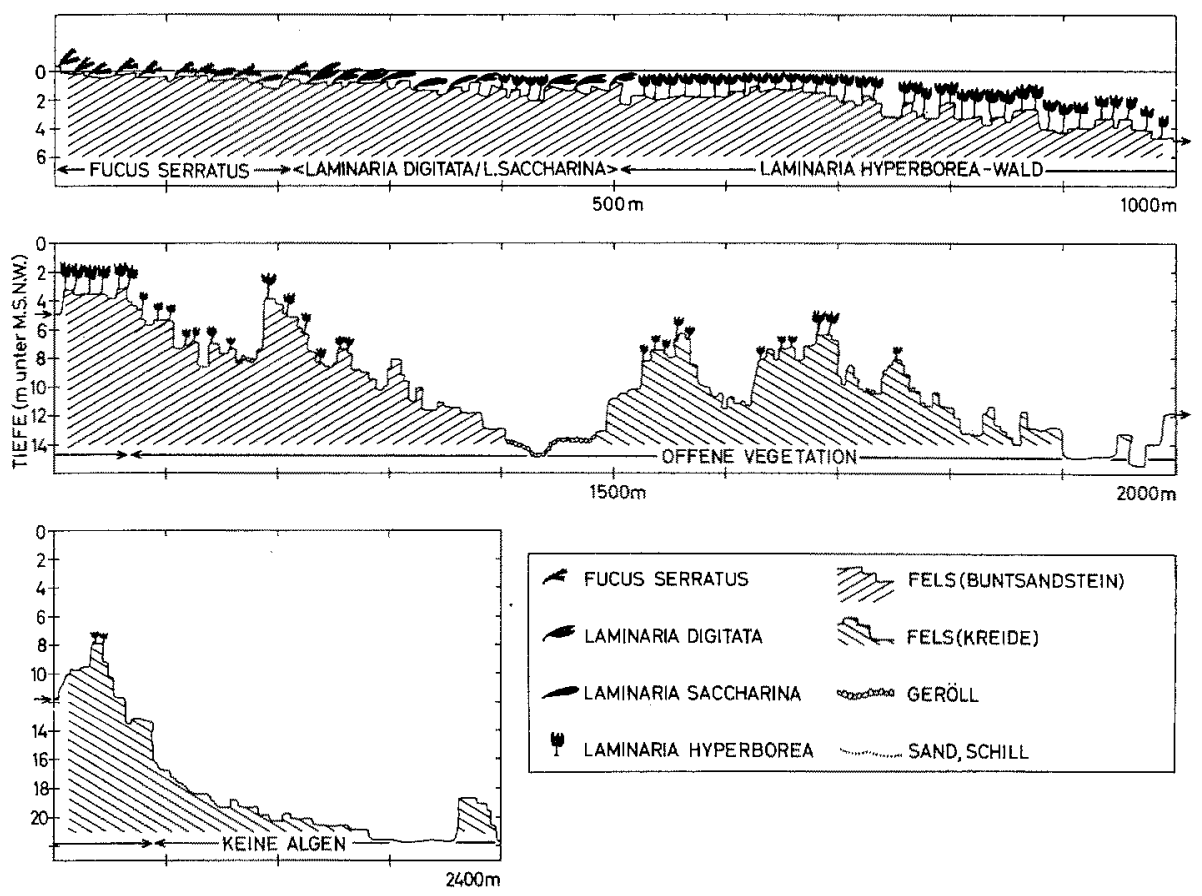

Abb. 8: Tauchprofil P 3 (im Nordwesten Helgolands; vgl. Abb. 1) mit schematsscher Darstellung der Verteilung der Brauntange, in Teilabschnitten von $0-1000 \mathrm{~m}, 1000-2000 \mathrm{~m}$ und $2000-2400 \mathrm{~m}$ dargestellt
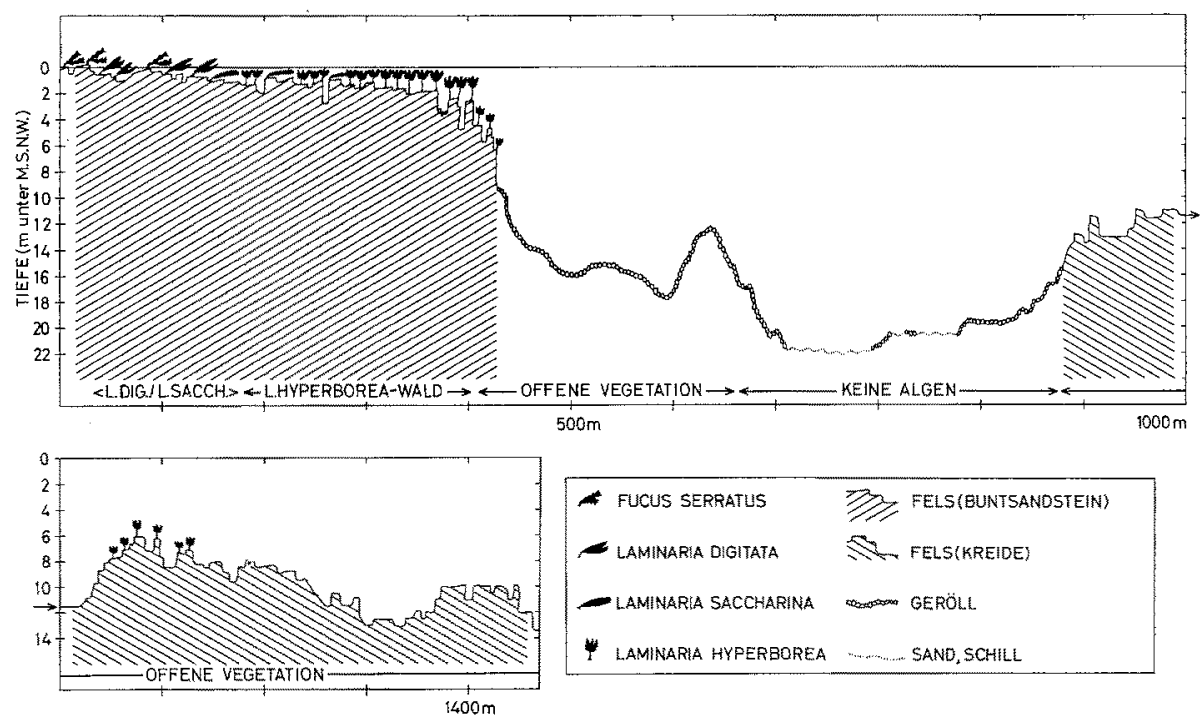

Abb. 9: Tauchprofil P 5 (im Südwesten Helgolands; vgl. Abb. 1) mit schematischer Darstellung der Verteilung der Brauntange, in Teilabschnitten von $0-1000 \mathrm{~m}$ und $1000-1450 \mathrm{~m}$ dargestellt 
eine steile, von etwa $4 \mathrm{~m}$ auf $10 \mathrm{~m}$ Tiefe abfallende Kante (Abb. 9, $430 \mathrm{~m}$ ). Darauf folgt das Geröllfeld des "Görtel" (Abb. 9, 430-980 m; Buntsandstein-, Hart- und Kreidegeröll), aus dem schließlich, in etwa $1 \mathrm{~km}$ Entfernung von der Uferschutzmauer West, die Kreideriffe des „Benners Rej" aufsteigen, die oberhalb von $8 \mathrm{~m}$ Tiefe wieder von Laminaria byperborea bewachsen sind (Abb. 9, 1050-1130 m).

\section{Die Vertikalverteilung der übrigen Algen}

Die Anzahl der längs der fünf untersuchten Tauchprofile in jeder Tiefenstufe aufgenommenen Quadrate geht aus Tabelle 1 hervor. Tabelle 2 enthält alle gefundenen Algenarten. Abbildung 10 zeigt die Tiefenverteilung von 38 Arten, die in mindestens acht der untersuchten 1232 Quadrate auftraten.

Durch Berechnung der prozentualen Häufigkeit in jeder Tiefenstufe wurde die Hauptverbreitungstiefe für jede der in Abbildung 10 dargestellten und nach zunehmender Hauptverbreitungstiefe angeordneten Arten ermittelt. Einige Arten sind in ihrem Vorkommen auf einen relativ eng umgrenzten Tiefenbereich beschränkt. So sind Cladophora rupestris (Nr. 8 in Abbildung 10) oder Dumontia incrassata (Nr. 9) nicht mehr unterhalb von $1,3 \mathrm{~m}$ Tiefe zu finden. Andererseits kommen Lomentaria clavellosa (Nr. 30), Lomentaria orcadensis (Nr. 33) oder Halarachnion ligulatum (Nr. 31) nur zwischen 4,5 und $8,5 \mathrm{~m}$ Tiefe vor. Ein ausgedehntes Tiefenspektrum zeigen Rbodomela confervoides (Nr. 20), Pbyllophora membranifolia (Nr. 21), Chaetomorpha melagonium (Nr. 22), Plocamium cartilagineum (Nr. 25), Polysiphonia urceolata (Nr. 27) und Ulva sp. (Nr. 29). Diese Arten wurden im Bereidh zwischen 0 und $4 \mathrm{~m}$ in Felsprielen auch auf nicht von Laminaria bewachsenen Steinen und Klötzen (58 untersuchte Quadrate je $25 \times 25 \mathrm{~cm}$ ) gefunden. Im gleichen Tiefenbereich, jedoch nur als Unterwuchs der geschlossenen Laminaria-Vegetation, kommen vor: Phycodrys rubens (Nr. 23), Delesseria sanguinea (Nr. 24), Pbyllopbora brodiaei (Nr. 28), Trailliella intricata (Nr. 32), Audouinella membranacea (Nr. 37) und Pbyllopbora crispa (Nr. 38). Diese Arten dringen weit in die Tiefe vor und vermögen im oberen Bereich des Sublitorals offensichtlich nur im Schatten des Phylloiddaches der Laminarien zu gedeiben.

Die zahlreichen Uberlappungen der Verteilungsspektren einzelner Arten (Abb. 10) erschweren die Zusammenfassung zu Verteilungsgruppen. Andererseits ändert sich der Aspekt der Vegetation bei zunehmender Wassertiefe in charakteristischer Weise. In Abbildung 11 wurde die Zusammensetzung der Vegetation (Sommeraspekt) in acht aufeinanderfolgenden Tiefenstufen grobschematisch dargestellt. In jeder Tiefenstufe wurden die Arten eingetragen, die in mindestens $5 \%$ aller in dieser Tiefenstufe untersuchten Quadrate vorkamen. Die Artnamen sind von links nach rechts entsprechend der abnehmenden Fundhäufigkeit aufgefürt. Bei der Betrachtung des Vegetationsaufbaus lassen sich mehrere Vegetationsschichten unterscheiden (Tab. 3). Die oberste Vegetationsschicht, das geschlossene Phylloiddach der Laminaria-Arten, ist nur zwischen 0,5 und $4 \mathrm{~m}$ Tiefe ausgebildet (Abb. 11, Tab. 3). Als nächste Vegetationsschicht kann die Epiphytenvegetation auf den Stielen von Laminaria byperborea angesehen werden. Hier handelt es sich vor allem um die beiden Arten Membranoptera 


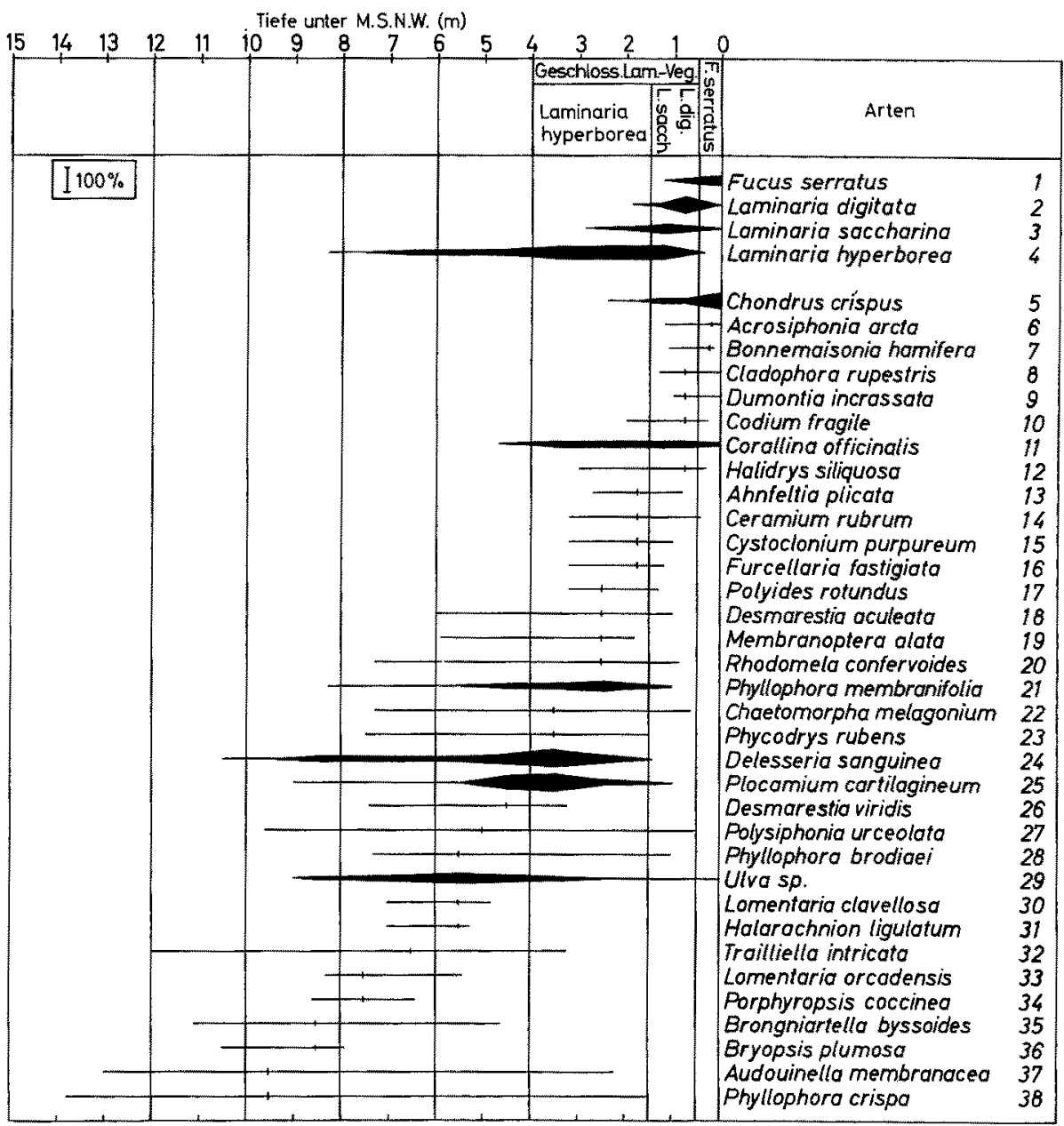

Abb. 10: Vertikalverteilung Helgoländer Algenarten im Sublitoral. Im oberen Teil des Diagramms sind die Tiefenbereiche der geschlossenen Vegetation von Fucus serratus, Laminaria digitata und Laminaria saccharina sowie von Laminaria byperborea angegeben. Die Vertikalverteilung der häufigsten Arten (Vorkommen in mehr als 10\% aller untersuchten Quadrate) ist quantitativ dargestell (Maßstab der relativen Häufigkeit für diese Arten in der linken oberen Ecke des Diagramms). Die Hauptverbreitungstiefe der übrigen Algen ist jeweils durch einen kurzen Vertikalstrich markiert. Vollständige Nomenklatur in Tabelle 2

Abb. 11 (rechts): Schematische Darstellung der Zusammensetzung der Helgoländer sublitoralen Vegetation (Sommeraspekt) in 8 aufeinanderfolgenden Tiefenstufen (Tiefe in Meter unter mittlerem Springniedrigwasser). Die Artnamen der Feinalgen sind in jeder Tiefenstufe, von links nach rechts gelesen, entsprechend der abnehmenden Fundhäufigkeit aufgeführt. Artnamen von Brauntangen, die oberhalb der Feinalgen ein geschlossenes Phylloiddach bilden, sind eingerahmt 


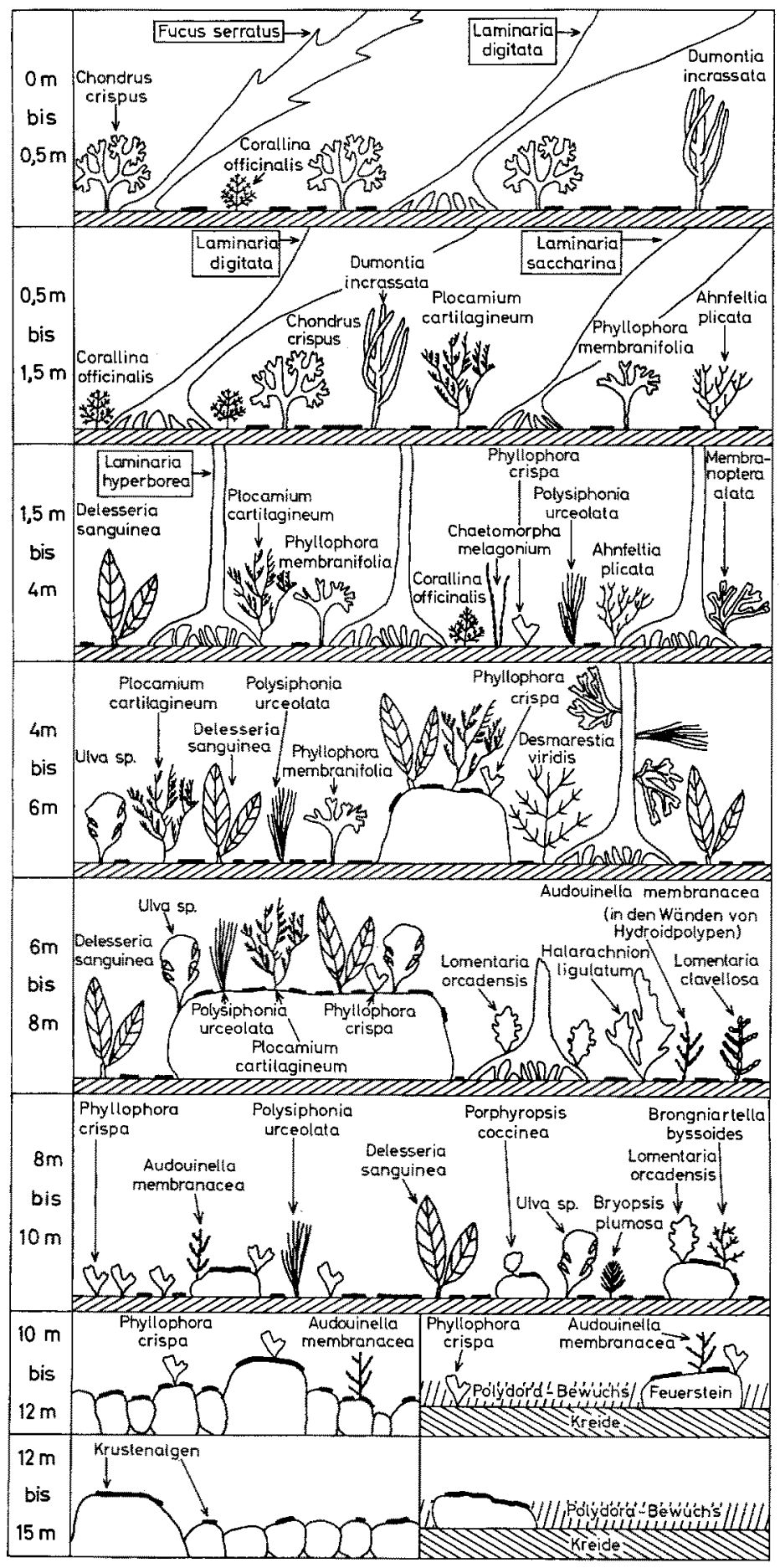


Tabelle 2

Aufstellung der bei Helgoland im Sublitoral gefundenen Algenarten (Nomenklatur nach PARKE \& DIXON 1968). Tiefenangaben in "Meter unter mittlerem Springniedrigwasser" Fundtiefe von seltenen Arten in Klammern

\begin{tabular}{|c|c|c|}
\hline Algenarten & Fundtiefe & $\begin{array}{l}\text { Nr. in } \\
\text { Abb. } 10\end{array}$ \\
\hline \multicolumn{3}{|l|}{ Rhodophyceae: } \\
\hline Abnfeltia plicata (Huds.) FRIEs & $0,8-2,7 \mathrm{~m}$ & 13 \\
\hline Antithamnion plumula (Ér.rs) THUR. in Le JoL. & $(6,0-7,0 \mathrm{~m})$ & - \\
\hline $\begin{array}{l}\text { Audouinella membranacea (MAGN.) PAPENF. } \\
=\text { Rbodochorton membranaceum }\end{array}$ & $2,2-13,0 \mathrm{~m}$ & 37 \\
\hline Bonnemaisonia hamifera HARIOT & $0,1-1,1 \mathrm{~m}$ & 7 \\
\hline Brongniartella byssoides (GOOD. et WOODW.) SchM. & $4,6-11,2 \mathrm{~m}$ & 35 \\
\hline Ceramium deslongchampsii CHAUV. in DUBY & $(0,0 \mathrm{~m})$ & - \\
\hline Ceramium rubrum (HUDs.) C. AG. & $0,4-3,2 \mathrm{~m}$ & 14 \\
\hline Chondrus crispus STACKH. & $0,0-2,4 \mathrm{~m}$ & 5 \\
\hline Corallina of ficinalis $\mathrm{L}$. & $0,0-4,7 \mathrm{~m}$ & 11 \\
\hline Cruaria sp. & & - \\
\hline $\begin{array}{l}\text { Cystoclonium purpureum (HuDs.) BATT. } \\
=\text { C } y \text { stoclonium purpurascens }\end{array}$ & $1,0-3,2 \mathrm{~m}$ & 15 \\
\hline Delesseria sanguinea (HUDs.) LAMOUR. & $1,4-10,6 \mathrm{~m}$ & 24 \\
\hline Dumontia incrassata (O. F. MÜLL.) LAMoUR. & $0,0-1,0 \mathrm{~m}$ & 9 \\
\hline Furcellaria fastigiata (L.) LAMOUR. & $1,2-3,2 \mathrm{~m}$ & 16 \\
\hline Gloiosiphonia capillaris (HuDS.) CARM. eX BERK. & $(7,0 \mathrm{~m})$ & - \\
\hline Gymnogongrus norvegicus (GUNN.) J. AG. & $(6,8 \mathrm{~m})$ & - \\
\hline Halaracbnion ligulatum (WOODW.) KüTz. & $5,3-7,0 \mathrm{~m}$ & 31 \\
\hline Hildenbrandia sp. & & - \\
\hline Lithothamnion sp. & & - \\
\hline Lithophyllum sp. & & - \\
\hline $\begin{array}{l}\text { Lomentaria clavellosa (TuRn.) GaILL. } \\
=\text { Chylocladia clavellosa }\end{array}$ & $4,8-7,0 \mathrm{~m}$ & 30 \\
\hline $\begin{array}{l}\text { Lomentaria orcadensis (HARv.) CoLL. eX TAYLOR } \\
\quad=\text { Lomentaria rosea }\end{array}$ & $5,5-8,3 \mathrm{~m}$ & 33 \\
\hline Membranoptera alata (Huds.) STACKH. & $1,8-5,9 \mathrm{~m}$ & 19 \\
\hline Petrocelis sp. & & - \\
\hline Peyssonnelia sp. & & - \\
\hline $\begin{array}{l}\text { Phycodrys rubens (L.) BaTT. } \\
\text { = Phycodrys sinuosa }\end{array}$ & $1,5-7,5 \mathrm{~m}$ & 23 \\
\hline Phyllophora brodiaei (TURN.) ENDL. & $1,0-7,4 \mathrm{~m}$ & 28 \\
\hline $\begin{array}{l}\text { Phyllophora crispa (HuDs.) DIXoN } \\
\quad=\text { Phyllophora rubens }\end{array}$ & $1,5-13,8 \mathrm{~m}$ & 38 \\
\hline Phyllophora membranifolia (Good. et Woodw.) J. AG. & $1,0-8,2 \mathrm{~m}$ & 21 \\
\hline Platoma bairdii (FARL.) KUCK. & $(7,0 \mathrm{~m})$ & - \\
\hline $\begin{array}{l}\text { Plocamium cartilagineum (L.) Dixon } \\
=\text { Plocamium coccineum }\end{array}$ & $1,0-9,0 \mathrm{~m}$ & 25 \\
\hline Plumaria elegans (BONNEM.) SсHм. & $(2,0 \mathrm{~m})$ & - \\
\hline Polyides rotundus (HuDs.) GREv. & $1,3-3,2 \mathrm{~m}$ & 17 \\
\hline Polysiphonia elongata (Huds.) SPRENG. & $(6,0 \mathrm{~m})$ & - \\
\hline Polysiphonia nigrescens (HuDS.) Grev. & $(0,0-0,5 \mathrm{~m})$ & $=$ \\
\hline Polysiphonia urceolata (LIGHTF. ex DILLW.) GREV. & $0,5-9,6 \mathrm{~m}$ & 27 \\
\hline Porphyropsis coccinea (J. Ag. ex AREsch.) Rosenv. & $6,5-8,6 \mathrm{~m}$ & 34 \\
\hline Rbodochorton floridulium (DILLW.) NÄG. & $(0,0 \mathrm{~m})$ & - \\
\hline $\begin{array}{l}\text { Rhodochorton purpureum (LIGHTF.) Rosenv. } \\
\quad=\text { Rhodocborton rothii }\end{array}$ & $(8,0 \mathrm{~m})$ & - \\
\hline $\begin{array}{l}\text { Rhodomela confervoides (HuDs.) SiLva } \\
\quad=\text { Rhodomela subfusca }\end{array}$ & $0,8-7,3 \mathrm{~m}$ & 20 \\
\hline $\begin{array}{l}\text { Trailliella intricata BATT. } \\
\quad=\text { Tetrasporophyt von Bonnemaisonia hamifera }\end{array}$ & $3,2-12,0 \mathrm{~m}$ & 32 \\
\hline
\end{tabular}


Tabelle 2 (Fortsetzung)

\begin{tabular}{|c|c|c|}
\hline Algenarten & Fundtiefe & $\begin{array}{l}\text { Nr. in } \\
\mathrm{Abb} .10\end{array}$ \\
\hline \multicolumn{3}{|l|}{ Phaeophyceae: } \\
\hline Chorda tomentosa LYNGB. & $(0,0-5,5 \mathrm{~m})$ & - \\
\hline $\begin{array}{l}\text { Cladostephus verticillatus (LiGHTE.) C. Ag. } \\
\text { Desmarestia aculeata (L.) LAMoUR. }\end{array}$ & $(6,0 \mathrm{~m})$ & $\overline{10}$ \\
\hline $\begin{array}{l}\text { Desmarestza aculeata (L.) LAMOUR. } \\
\text { Desmarestia viridis (O. F. MÜL.) LAMOUR. }\end{array}$ & $\begin{array}{l}1,0-6,0 \mathrm{~m} \\
3,2-7,4 \mathrm{~m}\end{array}$ & $\begin{array}{l}18 \\
26\end{array}$ \\
\hline Dictyota didhotoma (HuDs.) LAMOUR. & $(0,5 \mathrm{~m})$ & - \\
\hline Ectocarpus sp. & $(1,3-8,0 \mathrm{~m})$ & - \\
\hline Fucus serratus $\mathrm{L}$. & $0,0-1,2 \mathrm{~m}$ & 1 \\
\hline Giffordia sp. & $(0,5 \mathrm{~m})$ & - \\
\hline Halidrys siliquosa (L.) LYNGB. & $0,3-3,0 \mathrm{~m}$ & 12 \\
\hline Laminaria digitata (HuDs.) LAMOUR. & $0,0-1,9 \mathrm{~m}$ & 2 \\
\hline Laminaria byperborea (GUNN.) FosL. & $0,3-8,3 \mathrm{~m}$ & 4 \\
\hline Laminaria saccharina (L.) LAMOUR. & $0,0-2,9 \mathrm{~m}$ & 3 \\
\hline $\begin{array}{l}\text { Sphacelaria plumosa LYNGB. } \\
\quad=\text { Chatopteris plumosa }\end{array}$ & $(3,0-6,0 \mathrm{~m})$ & - \\
\hline Sphacelaria sp. & $(6,0-7,0 \mathrm{~m})$ & - \\
\hline Tilopteris mertensii (TurN. in SM.) KüTZ. & $(7,0 \mathrm{~m})$ & - \\
\hline \multicolumn{3}{|l|}{ Chlorophyceae: } \\
\hline Acrosipbonia arcta (DILLw.) J. Ag. & $0,0-1,2 \mathrm{~m}$ & 6 \\
\hline Bryopsis bypnoides LAMOUR. & $(0,0 \mathrm{~m})$ & - \\
\hline Bryopsis plumosa (Huds.) C. AG. & $7,9-10,6 \mathrm{~m}$ & 36 \\
\hline $\begin{array}{l}\text { Chatemorpha capillaris (KüTz.) BøRG. } \\
=\text { Chaetomorpha tortuosa }\end{array}$ & $(0,0-6,0 \mathrm{~m})$ & - \\
\hline Chaetomorpha melagonitum (WEB. et MOHR) KÜTZ. & $0,6-7,3 \mathrm{~m}$ & 22 \\
\hline Cladophora rupestris (L.) KüTz. & $0,0-1,3 \mathrm{~m}$ & 8 \\
\hline Codium fragile (SUR.) HARIOT & $0,2-2,0 \mathrm{~m}$ & 10 \\
\hline Enteromorpha sp. & $(0,0-6,0 \mathrm{~m})$ & - \\
\hline $\begin{array}{l}\text { Halicystis ovalis (LYNGB.) ARESCH. } \\
=\text { Gametophyt von Derbesia marina }\end{array}$ & $(6,0 \mathrm{~m})$ & - \\
\hline Monostroma sp. & $(0,2 \mathrm{~m})$ & $\ldots$ \\
\hline Ulva sp. & $0,0-9,0 \mathrm{~m}$ & 29 \\
\hline
\end{tabular}

Tabelle 3

Vorkommen von Vegetationsschichten in verschiedenen Tiefenbereichen im Helgoländer Sublitoral

\begin{tabular}{|lccccc|}
\hline Vegetationsschicht & $0,5-1,5 \mathrm{~m}$ & $1,5-4 \mathrm{~m}$ & $\begin{array}{c}\text { Tiefenbereich } \\
4-8 \mathrm{~m}\end{array}$ & $8-12 \mathrm{~m}$ & $12-15 \mathrm{~m}$ \\
\hline $\begin{array}{l}\text { Phylloiddach der ge- } \\
\text { schlossenen Lamina- } \\
\text { rienvegetation }\end{array}$ & $\times$ & $\times$ & - & - & - \\
$\begin{array}{l}\text { Stielpiphyten } \\
\begin{array}{l}\text { Feinalgen auf } \\
\text { Felsboden }\end{array}\end{array}$ & - & $\times$ & $\times$ & - & - \\
$\begin{array}{l}\text { Krustenalgen auf } \\
\text { Felsboden }\end{array}$ & $\times$ & $\times$ & $\times$ & $\times$ & - \\
\hline
\end{tabular}


alata und Polysiphonia urceolata. Im Tiefenbereich der geschlossenen Vegetation von L. byperborea, im sogenannten Laminarienwald, ist der Epiphytenbewuchs allerdings quantitativ nur schwach entwickelt; hier überwiegt der Bewuchs durch Epizoen (Bryozoen, Hydroidpolypen). Epiphyten gelangen erst an und unterhalb der unteren Grenze des Laminarienwaldes, bei $4 \mathrm{~m}$ Tiefe, an den Stielen von Laminaria byperborea zu üppiger Entwicklung. Die Stiele von Laminaria digitata und L. saccharina sind nur selten und nur im unteren Stielbereich, unmittelbar über den Haftkrallen, mit Epiphyten oder Epizoen besetzt. Epizoen (Membranipora membranacea) wachsen bevorzugt auf Phylloiden von $L$. byperborea, sehr selten auf den Phylloiden von $L$. digitata und L. saccharina. Mit Membranipora überwachsene Phylloide von L. byperborea tragen im Sommer auch schnellwüchsige Arten wie Ulva und Ceramium rubrum.

In der Vegetationsschicht der auf dem Felsboden wachsenden Feinalgen wurden zwischen 0 und $0,5 \mathrm{~m}$ Tiefe als häufigste Arten Chondrus crispus, Corallina officinalis sowie im Frühsommer Dumontia incrassata festgestellt (Abb. 11).

Im Unterwuchs der geschlossenen Vegetation von Laminaria digitata und $L$. saccharina (Abb. 11, Tiefenstufe 0,5 bis $1,5 \mathrm{~m}$ ) treten als häufige Algen Plocamium cartilagineum, Phyllophora membranifolia und Abnfeltia plicata hinzu.

Im Schatten des Laminarienwaldes ( $\mathrm{Abb}$. 11, Tiefenstufe 1,5-4 m) ist die Vegetationsschicht der Feinalgen quantitativ nur spärlich entwickelt. Delesseria sanguinea, Plocamium cartilagineum, Ulva sp. und Polysiphonia urceolata gelangen erst unterhalb des Bereichs des Laminarienwaldes zu üppiger Entfaltung (Abb. 11, Tiefenstufe 4-6 m) und herrschen auch noch in der Tiefenstufe zwischen 6 und $8 \mathrm{~m}$ vor. In diesem bzw. im folgenden Tiefenbereich $(8-10 \mathrm{~m})$ treten während der lichtreichen Jahreszeit, von Sommer bis Herbst, charakteristische Tiefen-Feinalgen, wie Lomentaria orcadensis, Lomentaria clavellosa, Halarachnion ligulatum, Brongniartella byssoides und Bryopsis plumosa als häufige Arten in Erscheinung.

Pbyllophora crispa und Audowinella membranacea repräsentieren die am tiefsten vordringenden Nicht-Krustenalgen im Helgoländer Sublitoral (Abb. 11, Tiefenstufe 10-12 m) und wurden sehr spärlich noch in $13,8 \mathrm{~m}$ bzw. $13 \mathrm{~m}$ Tiefe gefunden.

Die Vegetationsschicht der Krustenalgen überzieht den Felsboden im Bereich des gesamten Vorkommens der sublitoralen Vegetation (Tab. 3). Mit ihnen erreicht die Algenvegetation ihre untere Grenze (Abb. 11, Tiefenstufe 12-15 m). Mit bloßem Auge konnte bei Tauchgängen nur zwischen den verkalkten Gattungen Lithotbamnion und Lithopbyllum einerseits sowie andererseits den unverkalkten Formen unterschieden werden. Weitere Angaben sind auf Grund der Untersuchung gesammelter Steine möglich. Während Lithotbamnion und Lithophyllum vom unteren Eulitoral bis zur unteren Vegetationsgrenze vorkommen, scheinen. Hildenbrandtia sp. und Petrocelis sp., typische Formen im Eulitoral, nicht tief in das Sublitoral einzudringen. Peyssonnelia $s p$. ist vom unteren Eulitoral bis zur unteren Vegetationsgrenze verbreitet. Cruoria $s p$. überzieht unterhalb von etwa $6 \mathrm{~m}$ Tiefe häufig die gesamte Oberseite von Granitfindlingen, deren Seiten dicht von dem Polychaeten Pomatoceros triqueter bewachsen sind.

Auf den tieferen Kreideklippen, die von einem dichten Bewuchs des Polychaeten Polydora ciliata überzogen werden, sind die aufliegenden oder zum Teil noch in der Kreide steckenden Feuersteine das Hauptsubstrat für Krusten- und Feinalgen. Inmit- 
ten des dichten Rasens des Polychaeten Polydora ciliata wurden auf der Kreide lediglich kleine Exemplare von Phyllopbora crispa gefunden (Abb. 11, Tiefenstufe 10 bis $12 \mathrm{~m}$ ). Krustenalgen kommen auf dem Kreidefels nur vor, wo er nicht von Polydora ciliata besiedelt ist.

\section{DISKUSSION}

Die im Supra- und Eulitoral oft so scharfen Verteilungsgrenzen zwischen verschiedenen Arten und Artengruppen sucht man im Sublitoral vergebens, da das Licht, der wesentlichste Umweltfaktor, der die Vertikalverteilung sublitoraler Algen bestimmt, sich in seiner Quantität und Qualität kontinuierlich mit der Tiefe ändert. Es ist daher verständlich, daß über eine weitere begriffliche Aufgliederung des Sublitorals keine einheitliche Ansicht besteht. FELDMANN (1937) unterscheidet im Mittelmeer eine obere und eine untere Stufe des Sublitorals, „étage infralittoral supérieur" und „étage infralittoral inférieur"; in der vorliegenden Arbeit wurde aus den bei HaRTOG (1959) ausgeführten Gründen weiterhin der Begriff „Sublitoral“ statt „Infralitoral“ verwendet.

Als Grenze zwischen beiden Unterbereichen des Sublitorals wurde von FELDMANN (1937) die Tiefe angesehen, in welcher die lichtliebenden (photophilen) den schattenliebenden (sciaphilen) Arten weichen (Mittelmeer: 5-10 m Tiefe). Später schlug FELDMANN (1957) vor, eine Unterteilung des Sublitorals von den Ergebnissen weiterer Arbeiten abhängig zu machen. NEusHul (1965) unterscheidet zwar provisorisch drei Bereiche im Sublitoral ("upper sub-tidal", "middle sub-tidal", "lower sub-

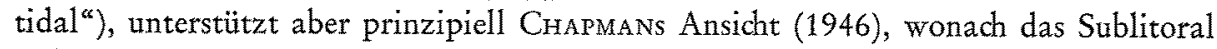
nicht unterteilt werden sollte, da die sublitorale Vegetation als Vegetationskontinuum erscheint. Diese Auffassung wird durch die Arbeit von Yosmid et al. (1963), durch eine spätere Untersuchung von Neushur (1967) sowie durch die Ergebnisse der vorliegenden Arbeit unterstïtzt.

Innerhalb des sublitoralen Vegetationskontiniuums können Gruppen von Arten mit ähnlichen Tiefenverteilungsspektren zwar auch als „Gemeinschaften“ aufgefaßt werden. Für den Bereich des Eulitorals an der niederländischen und französischen Kanalküste sowie bei Helgoland stellte HARTOG (1959) Algengemeinschaften auf. Alle Versuche, das sublitorale Phytal vegetationstypologisch zu gliedern, sind jedoch noch vorläufig. ScHWENKE (1969) warnt vor der unkritischen Übernahme terrestrisch-pflanzensoziologischer Methoden. Auch die vorliegende Arbeit geht davon aus, „es sei den spezifischen Verhältnissen des marinen Phytobenthals angemessener, zunächst mit vegetationsdeskriptiven Mitteln von betont allgemeinem Charakter zu arbeiten“ (SCHWENKE 1969, p. 83).

Bei Helgoland kommen Algen bis zu $15 \mathrm{~m}$ Tiefe vor, an der westschottischen Küste liegt die Grenze bei $33 \mathrm{~m}$ (McAllister et al. 1967), im Trondhjemsfjord bei $29 \mathrm{~m}$ (PRINTz 1926). Die geringe Tiefenausdehnung der Helgoländer sublitoralen Algenvegetation ist nicht etwa durch fehlendes festes Substrat unterhalb der Vegetationsgrenze bedingt, wie an vielen anderen Küsten, sondern sehr wahrscheinlich durch ein vergleichsweise schlechteres Lichtklima. Besonders von Oktober bis März, in der 
Tabelle 4

Tiefenverbreitung von Laminaria-Arten bei Helgoland und an der westschottischen Küste

\begin{tabular}{|lccc|}
\hline Fundort & L. digitata & L. saccharina & L. byperborea \\
\hline Helgoland & $0-2 \mathrm{~m}$ & $0-3 \mathrm{~m}$ & $0-8 \mathrm{~m}$ \\
Schottland (MCALLISTER et al. 1967) & $0-3 \mathrm{~m}$ & $0-18 \mathrm{~m}$ & $0-24 \mathrm{~m}$ \\
\hline
\end{tabular}

sturmreichen Jahreszeit, werden bei Helgoland nur geringe Transmissionswerte des Lichts gemessen (LüNING 1971).

Die tiefsten Exemplare von Laminaria byperborea wachsen bei Helgoland in $8 \mathrm{~m}$ Tiefe, an der schottischen Küste in $24 \mathrm{~m}$ Tiefe (vgl. Tab. 4). Laminaria digitata dagegen dringt auch an anderen Küsten nicht weit in das Sublitoral ein (KAIN 1962, SMITH 1967; Tab. 4). Laminaria saccharina, deren mehrjährige Vertreter bei Helgoland ebenfalls auf den oberen Bereich des Sublitorals beschränkt bleiben, kommt an der schottischen (KAIN 1962) und norwegischen Küste (PRINTZ 1926) noch in der Nähe der unteren Grenze von L. byperborea vor. Wie bereits erwähnt, zeigen junge Exemplare von $L$. saccharina auch bei Helgoland eine derartige Tiefenverbreitung, nicht jedoch mehrjährige Pflanzen.

In Tabelle 5 werden die unteren Tiefengrenzen einiger Arten an verschiedenen Küsten verglichen. Die Angaben von der westschottischen Küste gehen auf Tauchuntersuchungen zurück (McAllrster et al. 1967), die von der norwegischen Küste (Trondhjemsfjord) auf Dredschuntersuchungen (PRINTz 1926). Die große Anzahl der Arten mit einem weiten Tiefenspektrum wurde nicht berïcksichtigt. Vielmehr wurden die Arten ausgewählt, deren Tiefengrenzen einerseits etwa im oberen Drittel der gesamten Tiefenausdehnung der sublitoralen Vegetation liegen (Helgoland: $0-4 \mathrm{~m}$; schottische und norwegische Küste: $0-10 \mathrm{~m}$ ), andererseits etwa im unteren Drittel (Helgoland: $7-12 \mathrm{~m}$, unter Vernachlässigung des von Krustenalgen noch bewohnten Tiefenbereichs $z$ wischen 12 und $15 \mathrm{~m}$; schottische und norwegische Küste: $20-30 \mathrm{~m}$ ). Auf diese Weise ergibt sich am ehesten ein Hinweis für die Zugehörigkeit einzelner Arten zu den bereits von FELDMANN (1937) charakterisierten Gruppen der licht- und schattenliebenden (photophilen und sciaphilen) Arten. Es zeigt sich, daß zumindest an zwei, in einigen Fällen auch an drei Lokalitäten, die untere Tiefengrenze von Algen wie Chondrus crispus, Dumontia incrassata, Halidrys siliquosa schon im oberen Drittel des Sublitorals liegt, während zahlreiche Rotalgen, aber auch die Grünalge Bryopsis plumosa noch das untere Drittel erreichen. Die Vertikalverteilung einer Art läßt nicht unbedingt auf einen licht- oder schattenliebenden Charakter schließen. Vielmehr wäre experimentell zu prüfen, ob z. B. eine bestimmte Tiefenalge nur bei geringen Bestrahlungsstärken gedeihen kann, also empfindlich gegenüber höheren Bestrahlungsstärken ist, oder ob sie sowohl bei hohen als auch bei niedrigen Bestrahlungsstärken wächst. Die Angaben verschiedener Autoren über die oberen Vorkommensgrenzen sind in dieser Hinsicht oft nicht aussagekräftig, da viele Funde im oberen Drittel des Sublitorals aus der geschlossenen Laminaria-Vegetation stammen, wobei eine im Schatten des Phylloiddachs lebende Alge möglicherweise weniger Licht erhält als in größeren Tiefen, in denen das Phylloiddach der Laminarien fehlt. 
Tabelle 5

Vergleich der unteren Tiefengrenzen einiger Arten nach Untersuchungen an verschiedenen Küsten $(\mathrm{H}=$ Helgoland; $\mathrm{S}=$ Schottland; MCAllister et al. 1967; $\mathrm{N}=$ Norwegen, PRINTZ 1926)

\begin{tabular}{|c|c|c|c|c|c|c|}
\hline $\begin{array}{l}\text { Lokalität } \\
\text { Tiefengrenze zwischen }\end{array}$ & $\stackrel{\mathrm{H}}{\mathrm{O}-4 \mathrm{~m}}$ & $\stackrel{\mathrm{S}}{0-10 \mathrm{~m}}$ & $\begin{array}{c}\mathrm{N} \\
0-10 \mathrm{~m}\end{array}$ & $\begin{array}{c}\mathrm{H} \\
7-12 \mathrm{~m}\end{array}$ & $\stackrel{\mathrm{S}}{20-30 \mathrm{~m}}$ & $\stackrel{\mathrm{N}}{20-30 \mathrm{~m}}$ \\
\hline Chondrus crispus & $x$ & & $x$ & & & \\
\hline Cladopbora rupestris & $x$ & & $x$ & & & \\
\hline Dumontia incrassata & $x$ & $x$ & $x$ & & & \\
\hline Corallina of ficinalis" & $x$ & $x$ & & & & \\
\hline Halidrys siliquosa & $x$ & $x$ & $x$ & & & \\
\hline Abnfeltia plicata & $x$ & & $x$ & & & \\
\hline Furcellaria fastigiata & $x$ & $x$ & & & & \\
\hline Polyides rotundus & $x$ & $x$ & $x$ & & & \\
\hline Rbodomela confervoides & & & & $x$ & $x$ & $x$ \\
\hline Pbycodrys rubens & & & & $x$ & $x$ & $x$ \\
\hline Delesseria sanguinea & & & & $x$ & $x$ & $x$ \\
\hline Plocamium cartilagineum & & & & $x$ & $x$ & \\
\hline Desmarestis viridis & & & & $x$ & $x$ & \\
\hline Polysiphonia urceolata & & & & $x$ & $x$ & $x$ \\
\hline Phyllopbora brodiaei & & & & $x$ & & $x$ \\
\hline Ulva sp. & & & & $x$ & & $x$ \\
\hline Lomentaria clavellosa & & & & $x$ & $x$ & $x$ \\
\hline Trailliella intricata & & & & $x$ & & $x$ \\
\hline Lomentaria orcadensis & & & & $x$ & $x$ & \\
\hline Porphyropsis coccinea & & & & $x$ & & $x$ \\
\hline Brongniartella byssoides & & & & $x$ & $x$ & \\
\hline Bryopsis plumosa & & & & $x$ & $x$ & \\
\hline Audouinella membranacea* & & & & $x$ & & $x$ \\
\hline Phyllophora crispa & & & & $x$ & $x$ & \\
\hline *Vgl. Tabelle 2. & & & & & & \\
\hline
\end{tabular}

Unser Wissen über die Ursachen der Tiefenverbreitung einzelner Arten ist nicht zuletzt wegen der in der Vergangenheit oft unüberwindlichen technischen Schwierigkeiten noch dürftig. Untersuchungen über Lichtbedarf, Lichtresistenz, Abhängigkeit des Gasstoffwechsels von Lichtbedingungen und Temperatur sublitoraler Algen wären wünschenswert. Viele vegetationsphysiognomisch wichtige Arten sind in dieser Hinsicht bisher überhaupt nicht untersucht worden, da die Auswahl der Objekte in älteren Arbeiten oft nach rein praktischen Gründen erfolgen mußte. Wichtige Erkenntnisse lassen ferner Untersuchungen erwarten, die nur unter Einsatz der modernen Schwimmtauchmethode durchgeführt werden können, so etwa die Verpflanzung von gleichartigem Zuchtmaterial in verschiedene Tiefen, die Untersuchung des jahreszeitlichen Wachstums, die experimentelle Ermittlung des artspezifischen lichtabhängigen Uberlebensbereichs (Neushul \& Powell 1965) sowie langfristige, möglichst kontinuierliche Messungen des Unterwasserlichts in mehreren Tiefen und an verschiedenen Küsten.

\section{ZUSAMMENFASSUNG}

1. Die Vertikalverteilung der makroskopischen Algen im Helgoländer Sublitoral wurde an fünf Tauchprofilen von insgesamt $7200 \mathrm{~m}$ Länge untersucht. Weitere Tauchgänge wurden durchgeführt, um auch seltenere Arten zu erfassen. 
2. Zwischen 0,5 und $1,5 \mathrm{~m}$ Tiefe bilden Laminaria digitata und $L$. saccharina eine geschlossene Vegetation, zwischen 1,5 und $4 \mathrm{~m}$ L. byperborea. Mehrjärige Exemplare von $L$. digitata und $L$. saccharina kommen nicht unterhalb von $3 \mathrm{~m}$ Tiefe vor, L. byperborea wurde bis $8 \mathrm{~m}$ Tiefe gefunden.

3. Insgesamt wurden von 1965 bis 1970 im Helgoländer Sublitoral 66 Algenarten bzw. nicht weiter identifizierte Gattungen gefunden (Tab. 2). 38 Arten kamen in mehr als acht der untersuchten 1232 Quadrate $(25 \times 25 \mathrm{~cm}$ ) vor (Tiefenverteilung in $\mathrm{Abb}$. 10). Die sublitorale Algenvegetation erstreckt sich bei Helgoland bis $15 \mathrm{~m}$ Tiefe. Durch Vergleich mit Daten von anderen Küsten wurden zwei Artengruppen herausgestellt, deren untere Tiefengrenzen einerseits im oberen, andererseits im unteren Drittel des Sublitorals zu finden sind (Tab. 5).

Danksagungen. Herrn Prof. Dr. F. Gessner, Kiel, danke ich für die Anregung zu dieser Arbeit, Herrn Prof. Dr. O. Krnwe, Hamburg, für seine Initiative bei der Einführung des wissenschaftlichen Schwimmtauchens an der Biologischen Anstalt Helgoland sowie für sein großzügiges Entgegenkommen bei der Bereitstellung aller nötigen Arbeitsmittel. Für wertvolle Diskussionen, Ratschläge hinsichtlich der Durchführung der Arbeit bzw. hinsichtlich der Identifizierung von Algenmaterial danke ich Hern Dr. P. Kornmann, Helgoland, Herrn P.-H. Samling, Helgoland, Herrn Dr. H. Schwenke, Kiel, Herm Prof. P. Dixon, Irvine, USA, Herrn Prof. Dentzot, Paris, und Herrn Dr. T. Norton, Glasgow. Die Untersuchung wurde durch Mittel der Deutschen Forschungsgemeinschaft (Ki 41/10, Ki 42/21) unterstützt.

\section{ZITIERTE LITERATUR}

AleeM, A. A., 1956. Quantitative underwater study of benthic communities inhabiting kelp beds off California. Science, N. Y. 123, 183.

Chapman, V. J., 1946. Marine algal ecology. Bot. Rev. 12, 628-762.

Clarke, W. D. \& Neushux, M., 1967. Subtidal ecology of the Southern California coast. In: Pollution and marine ecology. Ed. by T. A. Olson \& F. J. Burgess. Wiley \& Sons, New York, 29-42.

ERnst, J., 1955. Sur la végétation sous-marine de la Manche d'après des observations en scaphandre autonome. C. r. hebd. Séanc. Acad. Sci., Paris 241, 1066-1068.

- 1959. Studien über die Seichtwasservegetation der Sorrentiner Küste. Pubbl. Staz. zool. Napoli 30, 470-518.

Feldmann, J., 1937. Recherches sur la végétation marine de la Méditerranée. La Côte des Albères. Revue algol. 10, 1-339.

- 1957. Les problèmes de l'étagement des peuplements d'algues marines. Colloques int. Cent. natn. Rech. scient. 1957, 37-41.

GISLEN, T., 1929/30. Epibioses of the Gullmar Fjord. K. svenska VetenskAkad. Skr. Naturskydd. $1929(3), 1-113 ; 1930(4), 1-380$.

HagmeIER, A., 1930. Die Besiedelung des Felsstrandes und der Klippen vor Helgoland. I. Der Lebensraum. Wiss. Meeresunters. (Abt. Helgoland) 15 (18 a), 1-35.

HARTOG, C. DEN, 1959. The epilithic algal communities occurring along the coast of the Netherlands. Wentia 1, 1-241.

KaIN, J. M., 1960. Direct observations on some Manx sublittoral algae. J. mar. biol. Ass. U. K. $39,609-630$.

- 1962. Aspects of the biology of Laminaria hyperborea. I. Vertical distribution. J. mar. biol. Ass. U. K. 42, 377-385.

- 1963. Aspects of the biology of Laminaria hyperborea. II. Age, weight and length. J. mar. biol. Ass. U. K. 43, 129-151.

- 1967. Populations of Laminaria byperborea at various latitudes. Helgoländer wiss. Meeresunters. 15, 489-499. 
KITching, J. A., 1941. Studies in sublittoral ecology. III. Laminaria forest on the West coast of Scotland; a study of zonation in relation to wave action and illumination. Biol. Bull. mar. biol. Lab., Woods Hole 80, 324-337.

- Macan, T. T. \& Gilson, H. C., 1934. Studies in sublittoral ecology. I. A submarine gully in Wembury Bay, S. Devon. J. mar. biol. Ass. U. K. 19, 677-705.

Konnas, J., 1959. Sea bottom vegetation of the Bay of Gdansk off Rewa. Bull. Acad. pol. Sci. Cl. II Sér. Sci. biol. 7, 5-10.

Kuckuck, P., 1897. Uber marine Vegetationsbilder. Ber. dt. bot. Ges. 15, 441-447.

Lamb, I. M. \& Zimmermann, M. H., 1964. Marine vegetation of Cape Ann, Essex County, Massachusetts. Rhodora 66, 217-254.

Larkum, A. W. D., Drew, E. A. \& Crossetr, R. N., 1967. The vertical distribution of attached marine algae in Malta. J. Ecol. 55, 361-371.

LüNING, K., 1969. Standing crop and leaf area index of the sublittoral Laminaria species near Helgoland. Mar. Biol. 3, 282-286.

- 1971. Seasonal growth of Laminaria byperborea under recorded underwater light conditions near Helgoland. In: Proceedings of the 4th European Marine Biological Symposium, Bangor, North Wales, U. K. (in press).

McAllister, H. A., Norton, T. A. \& Conway, E., 1967. A preliminary list of sublittoral marine algae from West of Scotland. Br. phycol. Bull. 3, 175-184.

McLEan, J. H., 1962. Sublittoral ecology of kelp beds off the open coast near Carmel, California. Biol. Bull mar. biol. Lab., Woods Hole 122, 95-114.

MrchaneK, G., 1967. Quantitative sampling of benthic organisms by diving on the Swedish west coast. Helgoländer wiss. Meeresunters. 15, 455-459.

Morgans, J. F. C., 1957. The benthic ecology of False Bay. I. The biology of infratidal rocks, observed by diving, related to that of intertidal rocks. Trans. R. Soc. S. Afr. 35, 387-442.

Neushul, M., 1965. Scuba diving studies of the vertical distribution of benthic marine plants. Botanica gothoburg. 3, 161-176.

- 1967. Studies of subtidal marine vegetation in Western Washington. Ecology 48, 83-94.

- \& Powel, H P., 1965. An apparatus for experimental cultivation of benthic marine algae. Ecology 45, 893-894.

NienburG, W., 1925. Die Besiedelung des Felsstrandes und der Klippen von Helgoland. II. Die Algen. Wiss. Meeresunters. (Abt. Helgoland) 15 (19), 1-15.

Norton, T. A., 1968. Underwater observations on the vertical distribution of algae at St. Mary's, Isles of Scilly. Br. phycol. Bull. 3, 585-588.

Parke, M. \& Dixon, P. S., 1968. Check-list of British marine algae-second revision. J. mar. biol. Ass. U. K. 48, 783-832.

PrinTz, H., 1926. Die Algenvegetation des Trondheimfjordes. Skr. norske Vidensk-Akad. (Mat.-nat. Kl.) 1926 (5), 1-273.

Rieds, R., 1967. Die Tauchmethode, ihre Aufgaben und Leistungen bei der Erforschung des Litorals; eine kritische Untersuchung. Helgoländer wiss. Meeresunters. 15, 294-351.

Schwenke, H., 1964. Vegetation und Vegetationsbedingungen in der westlichen Ostsee (Kieler Bucht). Kieler Meeresforsch. 20, 157-168.

- 1969. Meeresbotanische Untersuchungen in der westlichen Ostsee als Beitrag zu einer marinen Vegetationskunde. Int. Revue ges. Hydrobiol. 54, 35-94.

SMrth, R. M., 1967. Sublittoral ecology of marine algae on the North Wales coast. Helgoländer wiss. Meeresunters. 15, 467-479.

WAERN, M., 1952. Rocky-shore algae in the Oregrund Archipelago. Acta phytogeogr. suec. 30, 1-298.

Yoshida, T., SAWADA, T. \& Higaki, M., 1963. Sargassum vegetation growing in the sea around Tsuyazaki, north Kyushu, Japan. Pacif. Sci. 17, 135-143.

Anschrift des Autors:

Dr. K. LüNING

Biologische Anstalt Helgoland (Meeresstation)

2192 Helgoland

Deutschland (BRD) 